(1) 0.55 and 0.09 p. 100 .

(2) 0.68 and 0.09 p. 100 .

(3) 0.55 and 0.11 p. 100 .

(4) 0.68 and 0.11 p. 100.

Addition of tryptophan led to a marked increase in the feed intake (groups 3 and 4). Addition of lysine to a tryptophan deficient diet increased the sub-consumption of feed.

In two experiments ( $B$ and $C)$, 6-week old piglets $(11 \mathrm{~kg})$ receiving a tryptophan deficient and a tryptophan balanced diet ad libitum and in free choice, showed a clear preference for the second one; 87 and 74 p. 100 of the total feed intake in experiments $\mathrm{B}$ and $\mathrm{C}$, respectively. This difference was less marked in the case of a free choice between a lysine deficient and a lysine balanced diet.

In an experiment (D) conducted like the previous ones with weaned 6-week old piglets, the results obtained with an ad libitum feeding of the following 5 diets, were compared :

(1) Control diet containing 0.11 p. 100 tryptophan.

(2) (1) +0.03 p. 100 - L-tryptophan.

(3) (1) +0.06 p. 100 L-tryptophan.

(4) (1) +0.09 p. 100 L-tryptophan.

(5) (1) +0.12 p. 100 L-tryptophan.

The highest daily weight gain and the lowest feed conversion ratio were obtained with diet (3) containing 0.17 p. 100 tryptophan.

In the last experiment (E) carried out with the same basal diet as in the previous one, the effects of a supplementation with L-tryptophan $(0.03$ and $0.06 \mathrm{p}$. 100) and DL-tryptophan $(0.06$ and 0.12 p. 100$)$, were compared. As similar performances were obtained with diets supplemented with $0.06 \mathrm{p} .100 \mathrm{~L}$ or DL-tryptophan, it was concluded that the efficiency of D-tryptophan ranged around 100 p. 100.

\title{
Total replacement of soyabean in fattening pig diets by spring peas and synthetic tryptophan
}

\author{
M. PALISSE-ROUSSEL, L. JACQUOT, Y. MAURY \\ Sanders S.A., 17, quai de l'Industrie, 91260 Athis-Mons \\ avec la collaboration de la Société Mitsui \\ France
}

Total replacement of soyabean meal by spring peas $(37 \mathrm{p} .100)$ in a diet for fattening pigs based on maize $(50$ p. 100$)$ and wheat led during the "starter» period to significantly poorer growth rates and feed conversion ratios in both sexes and to a marked reduction of the feed intake in the females.

During the finishing period, the feed intake and performances were lower in females, while the results of males were similar to those obtained in the controls.

For the whole fattening period, growth and feed efficiency decreased by 10 and 7 p. 100 , respectively.

Supplementation of the "pea» diet with 0.03 or 0.06 synthetic tryptophan allowed, even with the lower level of incorporation, to restore a normal feed intake in the females. Performance, slightly lower during the first month, were improved during the finishing period, so that they were equivalent to those of the controls, which were excellent in absolute value. 